\title{
Spin Polarized Ground State for Interacting Electrons in Two Dimensions
}

\author{
Giuliano Benenti ${ }^{(a, b, c)}$, Gaëtan Caldara ${ }^{(a)}$, and Dima L. Shepelyansky ${ }^{(a)}$ \\ ${ }^{(a)}$ Laboratoire de Physique Quantique, UMR 5626 du CNRS, Université Paul Sabatier, 31062 Toulouse Cedex 4, France \\ ${ }^{(b)}$ International Center for the Study of Dynamical Systems, \\ Università degli Studi dell'Insubria, via Valleggio 11, 22100 Como, Italy \\ ${ }^{(c)}$ Istituto Nazionale di Fisica della Materia, Unità di Milano, via Celoria 16, 20133 Milano, Italy
}

(October 24, 2018)

\begin{abstract}
We study numerically the ground state magnetization for clusters of interacting electrons in two dimensions in the regime where the single particle wavefunctions are localized by disorder. It is found that the Coulomb interaction leads to a spontaneous ground state magnetization. For a constant electronic density, the total spin increases linearly with the number of particles, suggesting a ferromagnetic ground state in the thermodynamic limit. The magnetization is suppressed when the single particle states become delocalized.
\end{abstract}

PACS numbers: 71.10.-w, 75.10.-b, 75.50.Lk

Ferromagnetic instabilities result from the interplay between the electronic Coulomb interaction and the Pauli principle. In the Pauli picture, electrons populate the non-interacting orbitals of a system, such as a quantum dot or a metallic grain, in a sequence of spin up - spin down electrons. The resulting minimum spin state minimizes the kinetic energy: it costs energy to flip a spin since it must be promoted to a higher energy level. Thus the total spin of the system is $S=0$ when the number of electrons $N$ is even and $S=1 / 2$ at odd $N$. In contrast, the maximum spin allows a maximally antisymmetric coordinate wavefunction, thus reducing the effect of the Coulomb repulsion (a familiar example of this is Hund's rule for atoms). This leads to the Stoner instability [1], which gives a spontaneous magnetization when the typical interaction exchange energy between two particles close to the Fermi level is of the order of the single particle level spacing.

Spontaneous ground state magnetization gives rise to interesting effects, which are in the focus of many recent studies. In quantum dots, a ground state spin polarization can explain the absence of an even-odd asymmetry in the addition spectra in the Coulomb blockade regime [2]. Also, the addition of an electron to the dot may flip the spin of other electrons already in the dot: if the total spins of the ground states of successive number of electrons differ by more than $1 / 2$, spin selection rules suppress the corresponding conductance peak (spin blockade) [5]. Spontaneous magnetization effects could also explain the presence of kinks in the magnetic field dependence of the Coulomb blockade peak positions [6,7]. The stability of the minimum spin ground state in a quantum dot was analyzed for weak interactions in [8]. Within perturbation theory, the effective interaction strength is enhanced by the presence of disorder, leading to a ferromagnetic instability already below the Stoner threshold [9]. In the diffusive regime, recent studies have also considered the effect of mesoscopic wave function fluctuations 10 and of off-diagonal interaction matrix elements beyond the mean field treatment 11]. The appearance of local magnetic moments has been discussed also in the strongly correlated limit, at the quantum melting of the Wigner crystal 12 .

Although the Stoner instability signals the presence of short range magnetic ordering, it is not clear if it will also lead to a ferromagnetic ground state in the thermodynamic limit. Actually, the Stoner criterion is obtained within the mean field Hartree-Fock approximation and overestimates the long-range magnetic ordering, predicted also in one and two dimensions for the Hubbard model at finite temperatures, thus violating the Mermin and Wagner theorem [1]. The possibility of a ferromagnetic phase in strongly correlated two-dimensional (2D) systems was considered in 13, 14 (see also Refs. [15, 16]) and has recently received experimental support in dilute 2D electron gases [17]. At the same time, recent studies of fermionic models with random two-body interactions show that the ground state polarization is strongly reduced by off-diagonal interaction matrix elements [11, 18.

In this Letter, we investigate numerically the possibility of a ferromagnetic ground state in the regime where the single particle localization length is smaller than the system size. Without interaction this condition is always satisfied in two dimensions in the limit of large system size [19].

We study a disordered square lattice with $N$ fermions on $L^{2}$ sites. The Hamiltonian is defined by

$$
\begin{array}{r}
\hat{H}=-V \sum_{<\mathbf{i}, \mathbf{j}>, \sigma} c_{\mathbf{i} \sigma}^{\dagger} c_{\mathbf{j} \sigma}+\sum_{\mathbf{i} \sigma} \epsilon_{\mathbf{i}} n_{\mathbf{i} \sigma}+ \\
U_{H} \sum_{\mathbf{i}} n_{\mathbf{i} \uparrow} n_{\mathbf{i} \downarrow}+U \sum_{\mathbf{i} \neq \mathbf{j}, \sigma, \sigma^{\prime}} \frac{n_{\mathbf{i} \sigma} n_{\mathbf{j} \sigma^{\prime}}}{|\mathbf{i}-\mathbf{j}|},
\end{array}
$$

where $c_{\mathbf{i} \sigma}^{\dagger}\left(c_{\mathbf{i} \sigma}\right)$ creates (destroys) an electron at site $\mathbf{i}$ with spin $\sigma, n_{\mathbf{i} \sigma}=c_{\mathbf{i} \sigma}^{\dagger} c_{\mathbf{i} \sigma}$ is the corresponding occupation number, the hopping term $V$ between nearest neighbors characterizes the kinetic energy, random site energies $\epsilon_{\mathbf{i}}$ are taken from a box distribution over $[-W / 2, W / 2], U_{H}$ 
and $U$ measure the strength of the on-site Hubbard interaction and of the Coulomb interaction, respectively, and $|\mathbf{i}-\mathbf{j}|$ is the inter-particle shortest distance computed on a $2 \mathrm{D}$ torus (periodic boundary conditions are taken in both directions). In the following we choose $U_{H}=U$.

The Hamiltonian (11) commutes with the total spin $\left(\left[\hat{S}^{2}, \hat{H}\right]=0\right)$ and its component along an arbitrary $z$-direction $\left(\left[\hat{S}_{z}, \hat{H}\right]=0\right)$. Therefore $\hat{H}$ can be written in a block-diagonal form, with $N+1$ blocks where $S_{z}=-N / 2,-N / 2+1, \ldots, N / 2$, respectively. We consider the block with $S_{z}=0$ only, since it is sufficient for analysis of the ground state magnetization. Indeed, due to spin rotational symmetry, the system has a $2 S+1$ degeneracy (where $S$ is the total spin), with $S_{z}=-S, \ldots,+S$; therefore all the eigenvalues of the Hamiltonian (11) belong to the spectrum of the $S_{z}=0$ subspace.

The numerical studies of the model (1) at a finite density of interacting particles above a frozen Fermi sea are performed in the following way:

(i) Single particle eigenvalues $\epsilon_{\alpha}$ and eigenstates (orbitals $) \phi_{\alpha}(\mathbf{i})\left(\alpha=1, \ldots, L^{2}\right)$ are obtained via numerical diagonalization of the Hamiltonian (11) at $U_{H}=U=0$.

(ii) The Hamiltonian (1) is written in the basis of noninteracting orbitals obtained in (i):

$$
\begin{array}{r}
\hat{H}=\sum_{\alpha, \sigma} E_{\alpha} d_{\alpha \sigma}^{\dagger} d_{\alpha \sigma}+U_{H} \sum_{\alpha, \beta, \gamma, \delta} H_{\alpha \beta}^{\gamma \delta} d_{\alpha \uparrow}^{\dagger} d_{\beta \downarrow}^{\dagger} d_{\delta \downarrow} d_{\gamma \uparrow}+ \\
U \sum_{\alpha, \beta, \gamma, \delta, \sigma, \sigma^{\prime}} C_{\alpha \beta}^{\gamma \delta} d_{\alpha \sigma}^{\dagger} d_{\beta \sigma^{\prime}}^{\dagger} d_{\delta \sigma^{\prime}} d_{\gamma \sigma}
\end{array}
$$

with $d_{\alpha \sigma}^{\dagger}=\sum_{\mathbf{i}} \phi_{\alpha}(\mathbf{i}) c_{\mathbf{i} \sigma}^{\dagger}$, and transition matrix elements

$$
\begin{aligned}
H_{\alpha \beta}^{\gamma \delta} & =\sum_{\mathbf{i}} \phi_{\alpha}(\mathbf{i}) \phi_{\beta}(\mathbf{i}) \phi_{\gamma}(\mathbf{i}) \phi_{\delta}(\mathbf{i}) \\
C_{\alpha \beta}^{\gamma \delta} & =\sum_{\mathbf{i} \neq \mathbf{j}} \frac{\phi_{\alpha}(\mathbf{i}) \phi_{\beta}(\mathbf{j}) \phi_{\gamma}(\mathbf{i}) \phi_{\delta}(\mathbf{j})}{|\mathbf{i}-\mathbf{j}|} .
\end{aligned}
$$

(iii) The Fermi sea is introduced by restricting the sums in (2) to orbitals with energies above the Fermi energy $\epsilon_{M_{F}}: \alpha, \beta, \gamma, \delta>M_{F}$. We consider a filling factor $\nu_{F}=M_{F} / L^{2}=1 / 4$ (corresponding to $2 M_{F}$ frozen electrons due to spin degeneracy) and a finite density $\rho=N / L^{2}$ of $N$ interacting particles above the Fermi level. The frozen Fermi sea approximation is introduced for the sake of simplicity, since it allows us to avoid the band tail, where the single particle density of states and the one body localization length have a strong energy dependence. The advantages of such an approach have been demonstrated in 20. However, we have also checked that the results presented in this Letter are qualitatively similar when $M_{F}=0$.

(iv) The basis of the Slater determinants, built from the single particle orbitals $\phi_{\alpha}$, is energetically truncated at high energy orbitals by means of the condition
$\sum_{i=1}^{N}\left(m_{i}-M_{F}\right) \leq M$. Here $m_{i}$ is the orbital index for the $i$-th quasiparticle $\left(m_{i}>M_{F}\right)$. The truncated Hamiltonian still commutes with the total spin when $S_{z}=0$.

(v) We diagonalize the many-body truncated Hamiltonian. Then the total spin of a given eigenstates $\left|\Psi_{i}\right\rangle$ is found via the application of the operator $\hat{S}^{2}: \hat{S}^{2}\left|\Psi_{i}\right\rangle=$ $S_{i}\left(S_{i}+1\right)\left|\Psi_{i}\right\rangle$ (we take $\left.\hbar=1\right)$.

We consider $N=2,4,6,8$ particles on a square lattice of size $L=8,11,14,16$ respectively, at an approximately constant density $\rho=N / L^{2} \approx 1 / 32,0.5 \leq U / V \leq 2$, $2 \leq W / V \leq 10$. In particular, we focus on the localized regime $W=10 \mathrm{~V}$, where the single particle localization length $l_{1} \approx 4<L$. Data are averaged over a number of disorder configurations between 200 and 5000 .

The distribution of the energy differences $\delta E$ between the ground state energies $E_{0}(S)$ in spin sectors $S=1$ and $S=0$ is shown in Fig. 11 for $N=8$ particles in the localized regime with $W=10 \mathrm{~V}\left(\delta E=E_{0}(S=\right.$ $\left.1)-E_{0}(S=0)\right)$. One can see the mesoscopic Stoner mechanism: electron-electron interactions give a spontaneous magnetization $(\delta E<0)$, with a probability to have a polarized state increasing with the growth of interaction. The inset of Fig. 11 demonstrates that our results are stable when the size $N_{H}$ of the truncated Hilbert space is changed by a factor of five. Even though we cannot exclude the existence of very slow $N_{H}$-variations this check shows that the truncation does not significantly affect the ground state polarization.

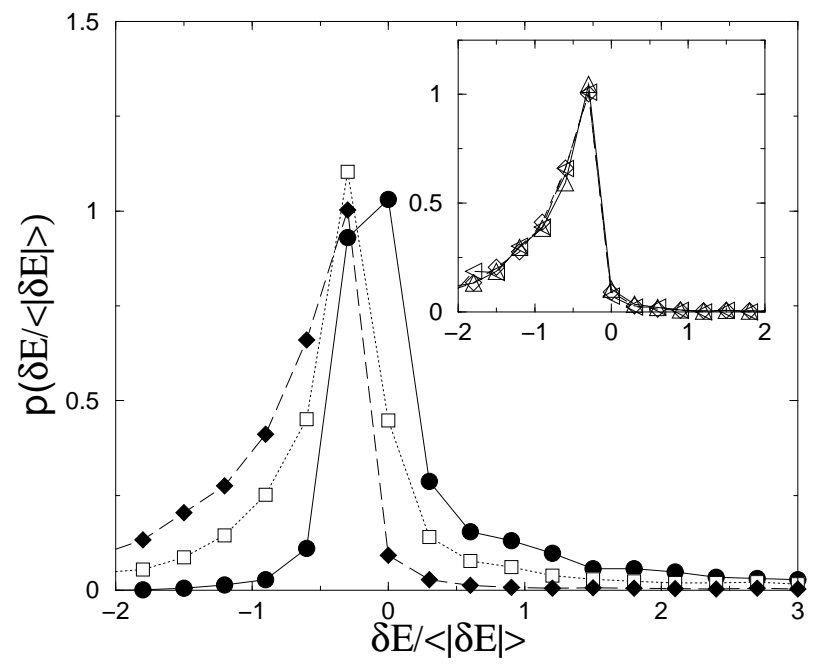

FIG. 1. Normalized distribution of the energy differences $\delta E$ between the ground state energies $E_{0}(S)$ in the spin sectors $S=1$ and $S=0$. Here $\delta E=E_{0}(S=1)-E_{0}(S=0)$ and $\langle|\delta E|>$ is the absolute value of $\delta E$ averaged over disorder configurations. Data are shown for $N=8$ particles on a square lattice of size $L=16$, disorder strength $W=10 \mathrm{~V}$, and interaction strengths $U=0.5 \mathrm{~V}$ (circles), $U=V$ (squares), and $U=2 V$ (diamonds). Inset shows the distribution at $U=2 \mathrm{~V}$ as a function of the size of the truncated Hilbert space: $N_{H}=932$ (triangles up), 2097 (diamonds), and 4354 (triangles left). 
The main result of our Letter is shown in Fig. 2; when the number of particle is increased (at a constant electronic density) the ground state can be found with high probability at larger and larger spin values. In the inset one can see that the ground state average magnetization increases linearly with the number of particles, $\langle S>\approx \alpha(U) N$ [21], with the slope $\alpha(U)$ growing with $U$, which determines the strength of the interaction exchange term. The extrapolation of the results presented in this figure would give a ferromagnetic ground state in the thermodynamic limit.

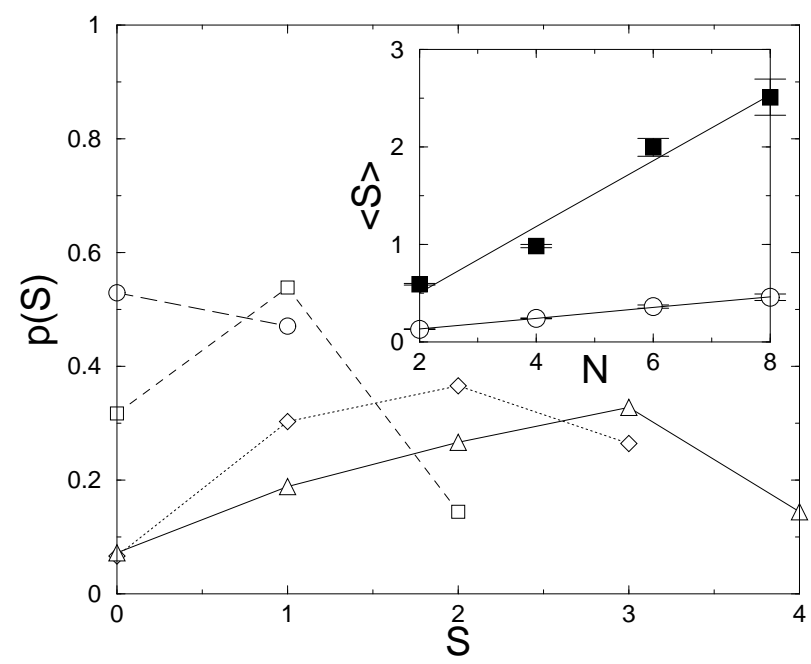

FIG. 2. Probability distribution $p(S)$ of the ground state spin for $W=10 \mathrm{~V}, U=2 \mathrm{~V}$. Here, $N=2, L=8$ (circles), $N=4, L=11$ (squares), $N=6, L=14$ (diamonds), $N=8, L=16$ (triangles). Inset: ground state average spin as a function of the number of particles for $U=0.5 \mathrm{~V}$ (circles) and $U=2 V$ (squares). Straight lines give linear fits, with $\langle S(U=0.5)>\approx 0.06 N$ and $<S(U=2)>\approx 0.34 N$. Here and in the next figures the error-bars show the size of statistical errors.

The dependence of the average magnetization on the disorder strength is shown in Fig.3. One can see that for sufficiently strong interaction disorder favors ground state spin polarization. Indeed, for $U / V=0.5$ at strong disorder, $W / V=10$, the total spin remains less than 0.5 while for $U / V=2$ it becomes five time larger. The significant average magnetization $\langle S\rangle$ appears in the localized single particle phase $(W / V=7,10)$, while in the delocalized regime $(W / V=2,4)$ it remains rather weak.

This is further confirmed in Fig. 1, which shows the size dependence of the average spin in the delocalized regime $W=2 V$. With the change of the number of particles between $N=2$ and $N=8$ the average magnetization $<S>$ remains constant, with non monotonous fluctuations around its average value. This is in a sharp contrast with magnetization behavior in the localized phase (see inset in Fig. 2) where $\langle S\rangle$ demonstrates a monotonous growth with $N$.

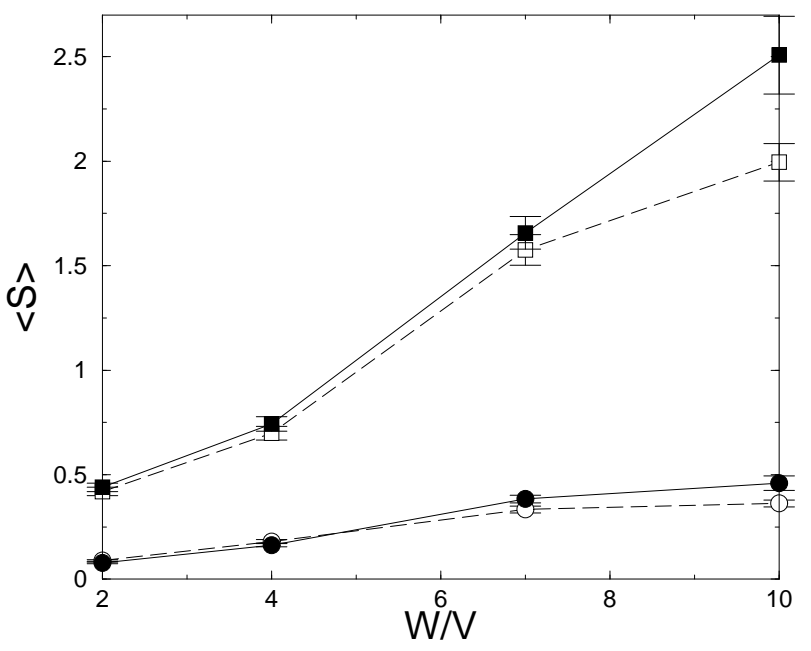

FIG. 3. Ground state average spin $\langle S\rangle$ as a function of disorder strength $W / V$, for $U=0.5 \mathrm{~V}$ (circles) and $U=2 \mathrm{~V}$ (squares); $N=6$ (empty symbols) and $N=8$ (filled symbols).

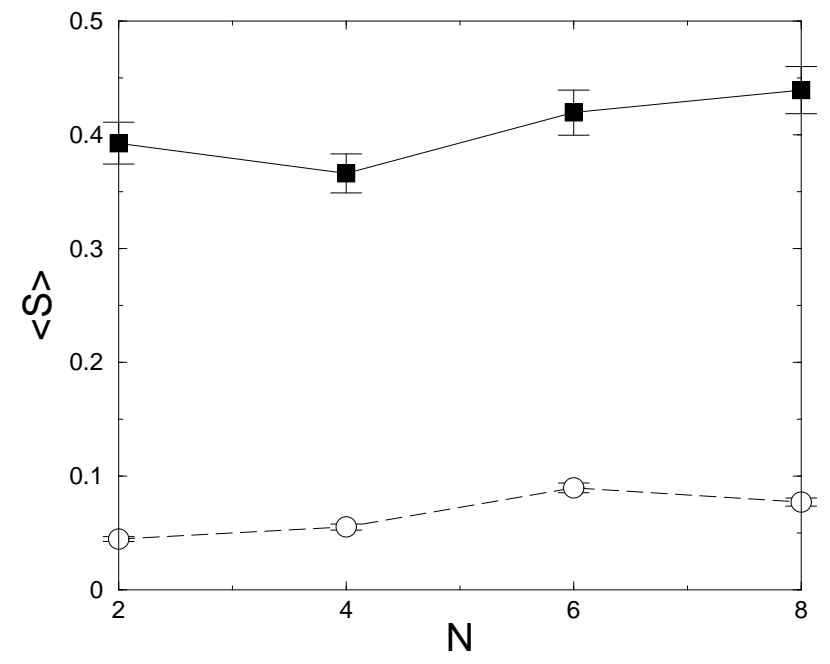

FIG. 4. Ground state average spin $\langle S\rangle$ as a function of the number of particles, for $W=2 \mathrm{~V}, U=0.5 \mathrm{~V}$ (circles) and $U=2 V$ (squares). The system size changes from $L=8$ for $N=2$ to $L=16$ for $N=8$.

The ensemble of our results allows us to propose the following physical scenario. In the regime when the Fermi energy is larger than the electron-electron interaction the perturbation theory [9] tells that the correction to the spin susceptibility $\delta \chi$ induced by interaction is inversely proportional to the conductance of the sample $g: \delta \chi \propto 1 / g$. With the increase of disorder $g$ drops and becomes of the order of one when the single particle localization length $l_{1}$ becomes comparable with the sample size. This indicates that the spin effects become more important in the regime of strong disorder, that is in agreement with our results (see Fig. 3). In the 
nonperturbative diffusive regime, non-diagonal interaction matrix elements start to give quantum fluctuations beyond mean-field Stoner approach. These interaction fluctuations favor small spin values, since the number of off-diagonal scattering events is larger in the lower spin sectors of the Hilbert space. This effect can prevent the ground state spin from achieving a full polarization [11]. On the contrary, in the localized regime the off-diagonal fluctuations are strongly reduced: due to single particle exponential localization, Coulomb repulsion can induce electron jumps only inside the localization domains, all the other scattering events giving an exponentially small contribution. Therefore, a possible scenario is the following: Stoner instability gives, at strong enough interaction and/or disorder, spin polarization in domains of the size of the single particle localization length, then the coupling between these domains gives global magnetization. The long range nature of the Coulomb interaction seems to play a crucial role in this physical picture. Indeed, recent quantum Monte Carlo studies of the ground state magnetization in the Hubbard model with disorder show disappearance of any magnetic order at strong disorder 22.

In summary, we have shown that Coulomb repulsion can lead to spontaneous ground state magnetization. In the regime with localized single particle wavefunctions, the total spin increases linearly with the number of particles. Even though we cannot exclude that this magnetic ordering can become limited at some large finite sizes, our results suggest the appearance of a ferromagnetic ground state induced by disorder and localization in the thermodynamic limit.

We thank the IDRIS in Orsay and the CalMiP in Toulouse for access to their supercomputers.

[1] A. Auerbach, Interacting Electrons and Quantum Magnetism (Springer-Verlag, New York, 1994).

[2] R. Berkovits, Phys. Rev. Lett. 81, 2128 (1998).

[3] S.R. Patel, S.M. Cronenwett, D.R. Stewart, A.G. Huibers, C.M. Marcus, C.I. Duruöz, J.S. Harris, Jr., K. Campman, and A.C. Gossard, Phys. Rev. Lett. 80, 4522 (1998).

[4] F. Simmel, D.Abusch-Magder, D.A. Wharam, M.A. Kastner, and J.P. Kotthaus, Phys. Rev. B 59, R10441 (1999).

[5] D. Weinmann, W. Häusler, and B. Kramer, Phys. Rev. Lett. 74, 984 (1995).

[6] H.U. Baranger, D. Ullmo, and L.I. Glazman, Phys. Rev. B 61, R2425 (2000).

[7] I.L. Kurland, R. Berkovits, and B.L. Altshuler, condmat/0005424

[8] O. Prus, A. Auerbach, Y. Aloni, U. Sivan, and R.
Berkovits, Phys. Rev. B 54, R14289 (1996).

[9] A.V. Andreev and A. Kamenev, Phys. Rev. Lett. 81, 3199 (1998).

[10] P.W. Brower, Y. Oreg, and B.I. Halperin, Phys. Rev. B 60, R13977 (1999).

[11] Ph. Jacquod and A.D. Stone, Phys. Rev. Lett. 84, 3938 (2000); cond-mat/0102100.

[12] F. Selva and J.-L. Pichard, cond-mat/0012015.

[13] M.J. Kelly and L.M. Falicov, Phys. Rev. Lett. 37, 1021 (1976).

[14] A.K. Rajagopal, S.P. Singhal, M. Banerjee, and J.C. Kimball, Phys. Rev. B 17, 2262 (1978).

[15] D. Varsano, S. Moroni, and G. Senatore, condmat/0006397

[16] B. Bernu, L. Cândido, and D.M. Ceperley, condmat/0008062

[17] S.A. Vitkalov, H. Zheng, K.M. Mertes, M.P. Sarachik, and T.M. Klapwijk, cond-mat/0009454

[18] L. Kaplan, T. Papenbrock, and C.W. Johnson, Phys. Rev. C 63, 014307 (2001).

[19] E. Abrahams, P.W. Anderson, D.C. Licciardello, and T.V. Ramakrishnan, Phys. Rev. Lett. 42, 673 (1979).

[20] X. Waintal, G. Benenti, and J.-L. Pichard, Europhys. Lett. 49, 466 (2000).

[21] The average spin $\langle S>$ is defined by the relation $\left\langle\left\langle\Psi_{i}\left|\hat{S}^{2}\right| \Psi_{i}\right\rangle\right\rangle=\langle S\rangle(\langle S\rangle+1)$, where $\langle\langle\ldots\rangle\rangle$ marks the averaging over the ground state and disorder configurations.

[22] M. Enjalran, F. Hébert, G. G. Batrouni, R. T. Scalettar, and S. Zhang, cond-mat/0102172. 